\title{
Integrated optimization and multi-agent technology for combined production and transportation planning
}

\author{
Jan A. Persson ${ }^{1}$ and Paul Davidsson ${ }^{2}$ \\ ${ }^{1}$ Blekinge Institute of Technology, Department Systems and Software Engineering, \\ 37424 Karlshamn, Sweden \\ ${ }^{2}$ Blekinge Institute of Technology, Department Systems and Software Engineering, \\ 37225 Ronneby, Sweden
}

\{Jan.Persson,Paul.Davidsson\}@bth.se

\begin{abstract}
In this research project, an integration of multi-agent technology and optimization techniques is suggested for the combined production and transport planning problem in a transport chain. The chain consists of a producer, a transport operator, and a number of customers. Optimization decomposition techniques use (dual) prices of resources to coordinate the generation of different plans in the so called sub-problems. We argue that these dual prices and generated plans can enhance the multi-agent based approach. By using the agent technology, we can better resemble the interactions between real planners reallocating resources. A transport chain within the food industry has been selected for validating the developed solution method.
\end{abstract}

\section{Introduction}

The integrated production and transportation planning problem is a complex problem. Particularly, this is the case with respect to the many resources to be coordinated and the number of planners/decision makers involved. The planning problem consists of a number of combinatorial decision problems: production scheduling (lot-sizing), fleet management and inventory management. Large benefits can be expected from a successful integration of these decision problems, which have been indicated by for example the use of VMI (Vendor Managed Inventory) approaches.

For a long time mathematical optimization techniques based on linear programming and branch and bound have been used to solve different types of resource allocation problems, e.g., production and transportation planning in various industries at strategic and tactical level, $[1,2,3]$. Additionally one can find examples of optimization techniques applied for short term planning (operational), e.g., activity scheduling $[4,5,6]$.

Solution approaches based on different optimization techniques are promising, particularly when used as heuristics for dynamic resource allocation problems. However, it is unlikely that these methods on their own will be the only route forward due to the high complexity and combinatorial aspects in this type of planning problems. Potentially constraint programming is an alternative to (classical) optimization techniques. The strength of constraint programming is the ability to handle combinatorial variables. However, it is less suitable for handling continuous variables representing, for example, inventory levels, production levels and transportation quantities. Further, constraint programming methods do not typically, in addition to use re-planning, encompass techniques for handling the dynamicity (i.e. frequent changes to the planning task). Stochastic optimization techniques may provide means for handling the dynamicity if useful information of the uncertainty is obtainable. However, since it is generally more difficult to use stochastic optimization problems, efficient re-planning may be a more efficient approach.

Agent-based computing has often been suggested as a promising technique for problem domains that are distributed, complex and heterogeneous [7]. The investigated problem in this research project, possess these characteristics. A number of agent-based approaches have been proposed to solve different types of resource allocation problems [8].

An agent-based approach supports the handling of dynamicity (by which it is implied that the planning situation may change rapidly and a response is required in a short amount of time). Further, the multi-agent based approach can support the situation when limited information is available for the planner to make decisions or plans. Moreover, the agent-based approach supports the designing of a solution method which encompasses the solution approach of real planners and dispatchers.

The above mentioned positive features of the multiagent based approach are typically not associated with or are at least not accentuated in optimization techniques.

According to a preliminary study [9], agent-based approaches tend to be preferable when:

- the problem domain is large

- the probability of node or link failures is high

- the time-scale of the domain is short

- the domain is modular in nature 
- the structure of the domain changes frequently

- there are sensitive information that should be kept locally

and mathematical optimization techniques when:

- the cost of communication is high

- the domain is monolithic in nature

- it is important that a system optimal (or near optimal) solution is found

- it is important that the quality of the solution can be guaranteed

We propose a solution method capitalizing on some of the relative merits of optimization techniques and some of the relative merits of agent-based approaches. In particular, we explore for the optimization techniques, the ability to achieve system optimality or near optimality with a quality assurance; and for the agent-based approach, we explore, the ability to handle a large problem domain and a short time-scale of the domain.

The proposed solution method utilizes the sub-problem solutions and dual prices obtained from a decomposition optimization techniques in order to strengthening an agent-based approach. The sub-problem solutions constitute potential good plans for the agents to implement. The dual prices constitute values of resources to be used in the cooperation between the agents for finding good solutions from a system perspective.

In this paper, we first describe the problem domain with some specifics of a studied transport chain in Section 2. In Section 3, we introduce the roles of the planners involved and map this to a rather generic agent-based description of the planning in a transport chain. The problem is formalized into an optimization formulation suitable for decomposition in Section 4. A decomposition based approach is outlined in Section 5. The suggested integration of the decomposition approach and the agentbased approach is outlined in Section 6 and conclusions are presented in section 7 .

\section{Problem Description}

The type of transport chain considered consists of one producer, a fleet of trucks, and a number of customers, se Figure 1.

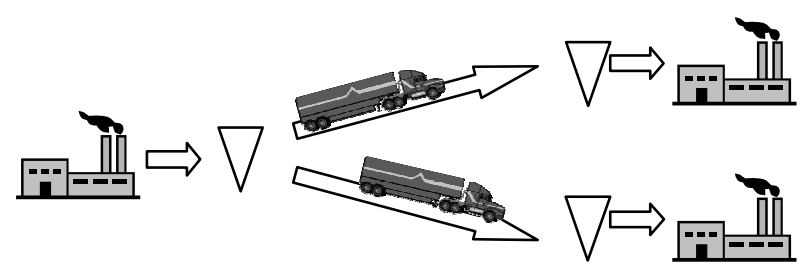

Figure 1 A transport chain consisting of a producer, a fleet of trucks, and customers.
The producer has limited production capacity and limited storage capacity of finished products. The producer can produce a number of different products. The transport operator uses a fleet of trucks for transporting the products to the customers. The trucks may also be used for other transports outside the studied transport chain when opportunities arise. However, the spot market is considered to be rather limited (due to the special requirements of food transports in the studied case). The transport operator may send its truck to one or more customers with respect to an outbound transport from the producer. At the customer, which may be a producer, there is often a limited inventory capacity available.

These characteristics of a transport chain can be found in a chain consisting of the producer Karlshamns AB, the transport operator FoodTankers, and a typical customer to Karlshamns AB, Procordia Foods. An important category of products produced at the Karlshamns AB is Oils \& Fats, which is mainly sold on the Scandinavia market. We focus on bulk products which typically are transported by FoodTankers. FoodTankers uses about forty-five trucks for transporting oils and fat products. The transport quantities to a particular customer are rarely less than 5 tons and rarely more than two customers are visited on an outbound trip from the producer.

In the studied transport chain, it is customer orders that drive the transportation and production planning. The customer orders in turn are based on the customer's production planning typically computed using MRP-logic, i.e. forecasted demand, available products and safety stock levels, lead time etc. At many customers of Karlshamns $\mathrm{AB}$, products may be stored before they are processed further.

Many of these customer orders to the producer are based on long-term (yearly) contracts of how much to order and to what prices. Further, the contracts include specifications of transport costs depending on the shipping quantities. Incitements for ordering full truck loads are typically included in the contracts. The contracts also specify a minimum lead time between order and expected delivery.

The production and transport planning has a rather short-term planning horizon (up to two weeks) and the part of the planning horizon normally considered for replanning is 2-4 days.

The (short-term) planning and re-planning is initiated by a customer orders. Currently the first planning step is to decide whether a new order can be accepted or not. If it is within the boundaries of contracts between producer and customer it is almost always accepted (else a penalty is incurred).

The subsequent production (re-)planning is about deciding when and in which order the products should be produced; and about planning the inventory storage. Lotsizing may apply by integrating different customer orders in the production. In this planning step, a backward 
scheduling is used respecting expected time required for delivery to the customers. Inventory storage capacities of finished products are rather limited and the products are often processed in the last production step at the same day as of delivery. Another motive for processing and deliver the products on the same day is requirements on the freshness of the product. However, no hard constraints for processing and deliver in the same day exist.

The transport planning concerns meeting the transport requirements specified by customer orders obtained via the producer. Currently not much flexibility exists with respect to altering the delivery times and quantities of transport orders. However, this may change partly as a result of this research project, which may show on the potential benefits from a system perspective of increasing this flexibility.

A challenge in the transport planning is to meet the requirements with existing transport capacity, without using overtime or hiring extra capacity. Further, the challenge is to explore additional transport options enabling a higher utilization of the trucks by increasing the loads when moving from customers "back" to the producer or locations close to the producer. When transport orders appear which require high cost solutions, the transport planner may suggest changes to the producer which in turn check for such changes with the customer. Such contacts may also be initiated when relatively small changes to the time and quantities of transport orders can deliver significant cost savings. However, currently it is rather hard to identify such potential cost savings.

The transport planning (fleet management) is carried out by a number of different planners each responsible of a particularly customer area. Each truck is normally assigned to such a customer area, but frequent temporary changes are done to this assignment. The transport planners are cooperating closely and are sitting in the same room with frequent contacts, which is helpful when reassigning truck to different customer areas.

\section{Roles for Planning in an Agent Perspective}

In an agent perspective, we have identified a number of important roles involved in the process of planning the transports and production. These are:

- Customer agent

- Transport chain coordinator agent

- Production planner agent

- Transport planner agent

These roles are connected to the transport chain as illustrated in Figure 2.

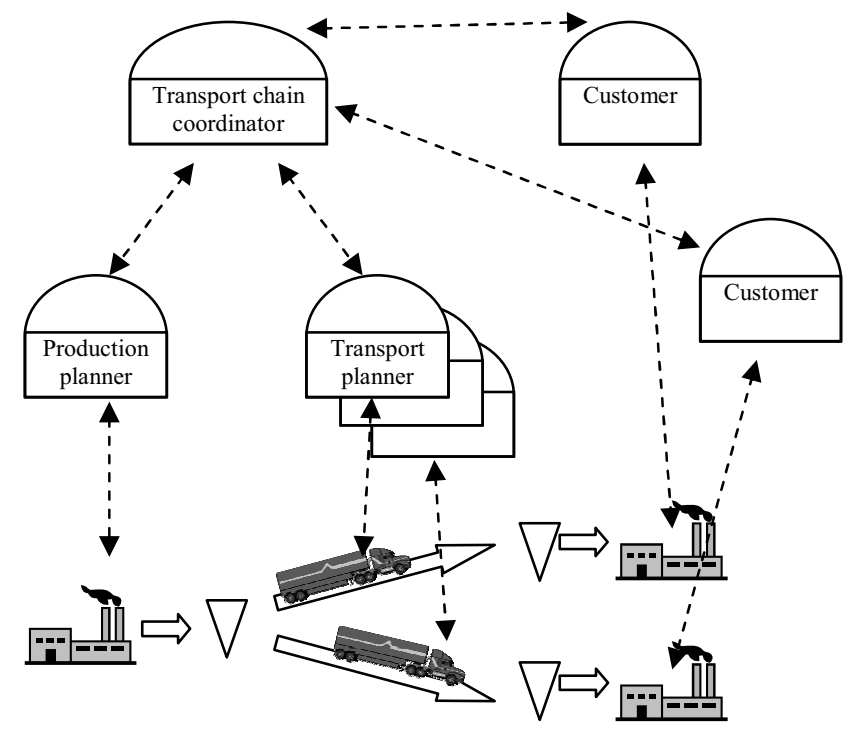

Figure 2 Decision making system of agents handling the transport chain.

This is in large a simplification of the general framework suggested in [10]. Here we consider a less general transport chain. Storage is only allowed at the producer or at the customer and only a single producer is considered. However, the transport planning of a single transport operator's fleet may here be handled by a number of agents and not just by a single agent.

We have included a number of agents for representing the transport planners each involved in the transport planning for a particular customer area. This may not be the case in a general setting of the problem.

The roles of the agents are further described in Table 1. 
Table 1 The roles of the agents in the decision system.

\begin{tabular}{|l|l|l|l|}
\hline $\begin{array}{l}\text { Decision } \\
\text { maker }\end{array}$ & $\begin{array}{l}\text { Decisions and } \\
\text { actions }\end{array}$ & Based on & Goal \\
\hline $\begin{array}{l}\text { Cus- } \\
\text { tomer } \\
\text { agent }\end{array}$ & $\begin{array}{l}\text { Makes requests } \\
\text { of products with } \\
\text { respect to quan- } \\
\text { tities, time of } \\
\text { delivery (or } \\
\text { time window). }\end{array}$ & $\begin{array}{l}\text { Anticipated cus- } \\
\text { tomer demand and } \\
\text { inventory levels at } \\
\text { the customer. }\end{array}$ & $\begin{array}{l}\text { Mediate cus- } \\
\text { tomer re- } \\
\text { quirements } \\
\text { including } \\
\text { costs for dif- } \\
\text { ferent deliv- } \\
\text { ery options. }\end{array}$ \\
\hline $\begin{array}{l}\text { Trans- } \\
\text { port } \\
\text { chain } \\
\text { coordi- } \\
\text { nator }\end{array}$ & $\begin{array}{l}\text { Decides how } \\
\text { much should be } \\
\text { shipped and } \\
\text { when. Makes } \\
\text { requests to pro- } \\
\text { duction and } \\
\text { transport plan- } \\
\text { ners. }\end{array}$ & $\begin{array}{l}\text { Requests from the } \\
\text { customer agents, } \\
\text { and on the an- } \\
\text { swers to the re- } \\
\text { quest of transpor- } \\
\text { tation and produc- } \\
\text { tion. }\end{array}$ & $\begin{array}{l}\text { Satisfy the } \\
\text { customer } \\
\text { requirements } \\
\text { at the lowest } \\
\text { possible sys- } \\
\text { tem cost. }\end{array}$ \\
\hline $\begin{array}{l}\text { Produc- } \\
\text { tion } \\
\text { planner }\end{array}$ & $\begin{array}{l}\text { Provides the } \\
\text { best offer to the } \\
\text { production re- } \\
\text { quest. Gives } \\
\text { production or- } \\
\text { ders to the pro- } \\
\text { ducer. }\end{array}$ & $\begin{array}{l}\text { Production capac- } \\
\text { ity, storage levels } \\
\text { at the production } \\
\text { site. }\end{array}$ & $\begin{array}{l}\text { Minimize } \\
\text { production } \\
\text { costs. }\end{array}$ \\
\hline $\begin{array}{l}\text { Trans- } \\
\text { port } \\
\text { planner }\end{array}$ & $\begin{array}{l}\text { Provides the } \\
\text { best offer to the } \\
\text { transport re- } \\
\text { quest. Assigns } \\
\text { to transport tasks } \\
\text { vehicles }\end{array}$ & $\begin{array}{l}\text { Transport capacity } \\
\text { and status (avail- } \\
\text { ability, position, } \\
\text { etc) of the trans- } \\
\text { port vehicles con- } \\
\text { trolled by the } \\
\text { operator. }\end{array}$ & $\begin{array}{l}\text { Minimize } \\
\text { transport } \\
\text { costs. }\end{array}$ \\
\hline
\end{tabular}

The production planner agent and transport planner agents should provide answers to the requests of the transport chain coordinator agent without implementing them. The transport chain coordinator agent will inform which requests and answers that should be implemented.

The suggested hierarchical design of the decision making system of agents allows for the study of different levels of cooperation. In an extreme, but rather common, case, the agents have pure local objectives (local cost minimizer) with virtually no sharing of information. In another extreme case, they are fully cooperative with the objective of minimizing total cost of the system. Since we want to improve the performance of the system by reducing total costs, we strive for the case when the agents are fully cooperative.

It is assumed that each agent has a plan of actions to take (currently and in the future). Then, inline with Table 1 , agents are cooperative and will accept a local increase of costs if it is assured that cost decreases as much or more in other parts of the system. Since we strive for system optimality it is important that the decision agents can handle rather complex offers implying changes to current plan. For example, the production planner may be given an offer to reallocate the production of a number of pro- duction orders to new dates for potentially achieving production plans which better match the transport requirements.

There are many possible mappings between organizations and the different decision making agents, see [10] for some examples. In the extreme case, all decision makers belong to the same organization for a transport chain, e.g., petroleum companies. Another extreme, is where all decision makers belongs to different organizations. Also, intermediate arrangements exist such as in the studied transport chain. In the studied transport chain, the transport chain coordinator agent and production planner agent are associated with the producing company. The customer agent and the transport planner agents are associated with the customer and the transport operating company, respectively. In the studied case, the function of the transport chain coordinator is mainly carried out by a customer order receiver, which cross-check for problems with production and transportation. In case of problems, e.g. hard to meet the customer request, the production planner and transport planner may cooperate directly for finding solutions. Although not common practice, the negotiations of changes to the customer orders (quantity and time of delivery) with the customer may also involve the production planner and transport planner directly.

\section{Optimization Model}

Next we formulate the integrated production and transportation planning problem. We choose the objective of minimize total cost including penalties for not meeting customer requests. Since the aim of this paper is not to explore solution methods for the details of the problems, such as lot-sizing and fleet management, the formulation is somewhat simplified. Thus, we will present the problem and a solution method at general level for illustrating the potential of integrating agent-based and decomposition approaches.

In order to formulate the problem we use the notation $p$ for a product in the set $P, t$ for a time period in the ordered set $T$, and $j$ for a customer in the set $J$. Further, we use the index $c$ for denoting such activities associated with a particular customer cluster $c \in C$, where $C$ is the set of customer clusters. The customers in a cluster $c$ are represented by the set $J_{c}$ and a customer $j$ belongs to a single such cluster. The problem can be formulated using the following variables:

- $\quad x_{p t}$, production of product $p$ in time period $t$,

- $\quad z_{j p t}$, quantity of product $p$ dispatched from the producer in time period $t$ to customer $j$.

- $\quad y_{j p t}$, quantity delivered to customer $j$ of product $p$ in time period $t$,

- $\quad o_{p t}$, quantity in storage at the producer of product $p$ in time period $t$,

- $\quad u_{j p t}$, quantity in storage at customer $j$ of product $p$ in time period $t$,

and the parameters: 
- $\quad d_{j p t}$, quantity demanded by customer $j$ of product $p$ in time period $t$.

The reason for introducing both the variables $z_{j p t}$, and $y_{j p t}$, is that the delivery to customer may occur in a time period later than the time period when the product was loaded into a truck at the producer. For convenience of notation we refer to the equivalent vector variables by dropping the indices. We introduce a general vector variable $v$, which represents the usage of trucks. We introduce this variable since the transport chain coordinator may consider a number of transport options. We avoid introducing an exact definition of the variable $v$ since this allows for different decomposition approaches introduced later.

We assume we have expressions of the cost functions according to:

- $\mathrm{f}^{\mathrm{l}}(x)$, production cost of producing the products at the producer,

- $\mathrm{f}^{2}(\mathrm{v}, y, z)$, transportation costs,

- $\mathrm{f}^{3}(o)$, inventory costs at the producer,

- $\mathrm{f}^{4}(u)$, inventory costs at the customers including penalty costs for not meeting the required demand.

Note that in order to compute these costs, a corresponding optimization problem may need to be solved, including additional variables and constraints, which we leave out for reasons of convenience.

We denote, for short hand notation, variables connected to a customer cluster $c \in C$, with the index $c$ according to $u_{c}, v_{c}, z_{c}$, and $y_{c}$. Here we assume the transportation costs represented by function $\mathrm{f}^{2}(v, y, z)$, can be separated for each customer cluster, such that, $\sum_{c \in C} f_{c}^{2}\left(v_{c}, y_{c}, z_{c}\right)=f^{2}(v, y, z)$. The same assumption applies for the separation of the cost function for inventories at the customers $\left(\mathrm{f}^{4}(u)\right)$.

Additionally we use the notation, $X, V, O$, and $U$ to denote the feasible set of the variables according to: $\mathrm{x} \in X$, $(v, y, z) \in V, o \in O, u \in U$.

The integrated transport and production planning problem can be formulated according to:

$$
\begin{array}{ll}
\min _{\mathrm{s.t.}}\left\{\mathrm{f}^{1}(x)+\mathrm{f}^{2}(\mathrm{v}, \mathrm{y}, z)+\mathrm{f}^{3}(o)+\mathrm{f}^{4}(u)\right\} & \\
o_{p, t-1}+x_{p t}-\sum_{j \in J} z_{j p t}=o_{p t} & p \in P, t \in T, \\
u_{j p, t-1}+y_{j p t}-d_{j p t}=u_{j p t} & j \in J, p \in P, t \in T, \\
x \in X, \quad(v, y, z) \in V, \quad o \in O, u \in U . &
\end{array}
$$

The constraints (1) model the inventory balance between time periods at the producer of the different products. In constraints (2), the equivalent inventory balances at the customers are modeled.

The sets, $X, V, O$, and $U$, may contain rather complex restriction on the variables. Further, they may implicitly include additional variables representing issues associated with production, storage, and transportation. However, these issues can be seen as hidden in separate parts representing production, transportation, and consumption with the feasible sets $X, V, O$, and $U$. The set $\mathrm{X}$ may contain restriction and variables handling the sequencing of production of different products. An important issue is how the transportation $(v)$ is modeled and connected to dispatching (z) and delivery (y). For instance, the set $V$ connects the quantities delivered from the producer to the customer which may contain a model of the possible routes for the trucks. In the feasible set $U$ (inventory levels at the customer) and in the associated cost $\mathrm{f}^{4}(u)$, penalties for not meeting inventory requirements and costs associated with deliveries at non-preferred dates and times may be included.

\section{Decomposition}

Decomposition of optimization problems is generally motivated by the algorithmic performance of the approach. Specific motives can, for example, be connected to strengthening the linear relaxation, to handle a large problem in terms of variables and constraints, or to generate good solutions heuristically.

For a given optimization problem there are typically a number of ways to design the decomposition. It is crucial to choose a suitable decomposition approach for achieving algorithmic efficiency. In the present work, the main motive is to outline how the elements of the decomposition can resemble the roles and agents identified above and to get valuable input to how the different agents may cooperate in an efficient way. For further reading on decomposition we suggest $[1,11]$.

A possible decomposition approach is in effect to Lagrange relax constraints (1), and (2), using Lagrange multipliers $\mu$, and $\lambda$, respectively. In the following we refer to the multipliers as dual prices since we view them in a Dantzig-Wolfe decomposition setting. By relaxing these two groups of constrains, a sub-problem for the production, a sub-problem for transportation to each customer cluster $c \in C$, and customer inventory sub-problem, can be obtained. The following problem represents the producers problem:

$$
\begin{aligned}
& \min \left\{\mathrm{f}^{\mathrm{l}}\left(x_{c}\right)+\mathrm{f}^{3}\left(\mathrm{o}_{\mathrm{c}}\right)+\sum_{p \in P} \sum_{t \in T}\left(\mu_{p t}\left(o_{p, t-1}+x_{p t}-o_{p t}\right)\right)\right\} \\
& \text { s.t. } \quad x \in X, \quad o \in O .
\end{aligned}
$$

For each customer cluster $c \in C$, we have a transport optimization sub-problem: 


$$
\begin{aligned}
& \min \left\{\mathrm{f}_{\mathrm{c}}^{2}\left(\mathrm{v}_{\mathrm{c}}, y_{c}, z_{c}\right)-\sum_{j \in J_{c}} \sum_{p \in P} \sum_{t \in T} \mu_{p t} z_{j p t}+\right. \\
& \left.\sum_{j \in J_{c}} \sum_{p \in P} \sum_{t \in T} \lambda_{j p t} y_{j p t}\right\} \\
& \text { s.t. } \quad\left(v_{c}, z_{c}, y_{c}\right) \in V_{c} .
\end{aligned}
$$

For each customer cluster $c \in C$, we have a customer inventory sub-problem:

$$
\begin{aligned}
& \min \left\{\mathrm{f}_{\mathrm{c}}^{4}(u)+\sum_{j \in J_{c}} \sum_{p \in P} \sum_{t \in T} \lambda_{j p t}\left(u_{j p, t-1}-u_{j p t}\right)\right\} \\
& \text { s.t. } \quad u_{c} \in U_{c}
\end{aligned}
$$

An important and central part in the problem above, is the part modeling the transports including dispatching $(\mathrm{z})$ and delivery (y), that is the set $V$. Here, we suggest a rather rudimentary, but concrete, modeling of the transportation problem. For this purpose we introduce the variable $v_{c t}$, which is the number of trucks allocated to customer cluster $c$ in time period $t$ and parameter $e_{\mathrm{t}}$, which represents the number of trucks available in time period $t$. In addition, we use $g$ to denote the truck capacity (here assumed to be equal for all trucks). Further, we introduce an indicator, $\xi_{j}$, representing the number of time periods required for transportation between dispatching at the producer and delivery to a customer $j$. Then we can consider constraint:

$\sum_{\mathrm{c} \in \mathrm{C}} v_{\mathrm{ct}}<=e_{t} \quad t \in T$

and the optimization problem

$$
\begin{aligned}
& \min \left\{\mathrm{f}^{1}(x)+\mathrm{f}^{2}(\mathrm{v}, \mathrm{y}, z)+\mathrm{f}^{3}(o)+\mathrm{f}^{4}(u)\right\} \\
& \text { s.t } \quad(1),(2) \text { and }(3),
\end{aligned}
$$

which constitutes the base for the coordinating problem (or master problem), where the Lagrange multiplier $\tau$ is used for constraint (3).

For each customer cluster $c \in C$, we now have the transport optimization sub-problem:

$$
\begin{gathered}
\min \left\{\mathrm{f}_{\mathrm{c}}^{2}\left(\mathrm{v}_{\mathrm{c}}, y_{c}, z_{c}\right)-\sum_{j \in J_{c}} \sum_{p \in P} \sum_{t \in T} \mu_{p t} z_{j p t}+\right. \\
\left.\sum_{j \in J_{c}} \sum_{p \in P} \sum_{t \in T} \lambda_{j p t} y_{j p t}+\sum_{t \in T} \tau_{t} v_{c t}\right\} \\
\text { s.t. } \quad \mathrm{z}_{j p t}=\mathrm{y}_{\mathrm{jp}, \mathrm{t}+\xi_{j}} \quad j \in J, p \in P, t \in T, \\
\sum_{j \in J_{c}} \sum_{p \in P} z_{j p t} \leq g v_{c t} \quad t \in T, \\
\left(v_{c}, z_{c}, y_{c}\right) \\
\in V_{c} .
\end{gathered}
$$

In the above sub-problems the constant contributions to the objective functions have been ignored $\left(e_{t} \tau\right)$, since these contributions do not affect the plans generated, represented by variables: $x, o, v, z, y, u$. However, in a Dantzig-Wolfe decomposition, these contributions must be accounted for when deciding on whether to terminate the solution procedure or not.

In this rudimentary case, the set $V_{c}$ may simply include non-negativity constraints on the variables and integer constraints on $v_{c t}$. In a more detailed representation of the transportations, a set of truck schedules can be pregenerated and from which each truck has to select such a schedule in each time period. Additionally one may use decomposition approach where schedules are generated for the trucks in the sub-problems and the selection of which schedule to use is made in the coordination problem (see [12] for a similar approach). For illustration purpose, we assume that the transportation problem is modeled rudimentary as suggested above in the following.

Figure 3 outlines the different optimization problems and the information sent between the sub-problems and a coordination problem. The coordination problem and the sub-problems are solved interchangeably (all the subproblems in parallel). The role of the coordination problem (the restricted master problem) is to find the best solution given the sub-problem solutions generated so far. While doing so, it will achieve its most important role: to generate new dual prices to be communicated to the subproblems.

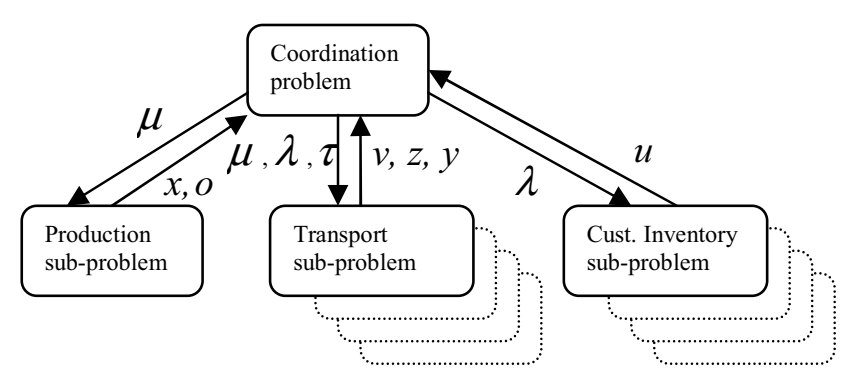

Figure 3 Outline of the information sent in a decomposition approach of the considered problem.

In our application, we have assumed that the customer's production plan has been fixed, and we are considering the customer demand $d_{j p t}$ as a parameter. This implies that there is little flexibility in the customer inventory sub-problem; it is only the inventory levels that can be planned. Then it might be useful to consider the customer inventory levels together with transportation planning. In such case, for each customer cluster $c \in C$, we have a transport and customer inventory optimization subproblem: 


$$
\begin{gathered}
\min \left\{\mathrm{f}_{\mathrm{c}}^{2}\left(\mathrm{v}_{\mathrm{c}}, y_{c}, z_{c}\right)+\mathrm{f}_{\mathrm{c}}^{4}(u)-\sum_{j \in J_{c}} \sum_{p \in P} \sum_{t \in T} \mu_{p t} z_{j p t}+\right. \\
\left.\sum_{j \in J_{c}} \sum_{p \in P} \sum_{t \in T} \lambda_{j p t}\left(u_{j p, t-1}+y_{j p t}-u_{j p t}\right)+\sum_{t \in T} \tau_{t} v_{c t}\right\} \\
\text { s.t. } \quad j \in J, p \in P, t \in T, \\
\sum_{j p t}=\mathrm{y}_{\mathrm{jp}, \mathrm{t}+\xi j} \sum_{j \in J_{c}} z_{p \in P} \leq g v_{c t} \quad t \in T, \\
\left(v_{c}, z_{c}, y_{c}\right) \in V_{c}, u_{c} \in U_{c .} .
\end{gathered}
$$

In Figure 4, the case of combined transport and customer inventory sub-problems are outlined.

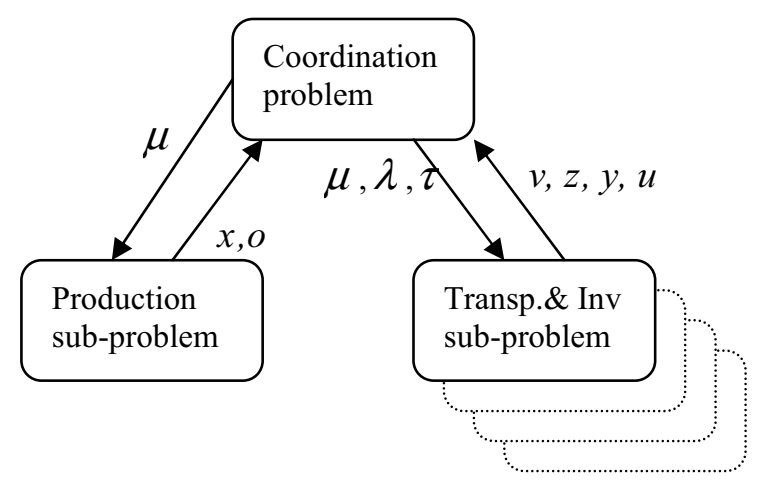

Figure 4 Outline of the information sent in a decomposition approach in case of combined transport and customer inventory sup-problems.

Figures 3 and 4 represent the solution procedure needed for solving a type of linear relaxation to the problem. The coordination problem (e.g. a Dantzig-Wolfe restricted master problem formulation) and the subproblems will iteratively be solved until the process converges, i.e. until no improving solutions can be found. The coordination problem provides new updated dual prices $\mu, \lambda$, and $\tau$, based on previous values of variables for production, transportation, and inventory levels (i.e. based on sub-problem solutions generated).

In the production sub-problem, a production plan is developed given the values of inventories of products in different time periods, i.e. given by $\mu$. Hence the production planning is simplified compared to the real production planning problem since in the sub-problem the exact quantities of transports can be ignored. In the real production planning problem, the transport quantities are given by backward scheduling of customer orders. Issues of sequencing and start-ups of production may be accounted for in the sub-problem by introducing them in the feasible set $X$.
In the transport sub-problem, transports are considered and explicit routing of the trucks can be considered. It is simplified compared to real-life transport planning and fleet management, since the quantities to transport are not dictated by the production and customer requirements explicit, but by current values/costs of the products in different time periods, i.e. the values $\mu$ and $\lambda$. The values of $\tau$, represent cost for using trucks in different time periods. In this sub-problem a number of rules concerning the trucks and drivers, such as, rules for drivers driving hours can be included in the feasible set V.

The customer inventory sub-problem is a rather trivial problem, when the demand is fixed. It is about deciding optimal inventory levels with respect to values of inventory levels at different time periods and inventory constraints. Alternatively, this can be accounted for in an integrated transport and customer inventory sub-problem, as suggested above.

In order to solve this problem using the above scheme for Dantzig-Wolfe decomposition, a strategy for handling the combinatorial aspects is typically required. A single production and a single transport plan for each customer must be chosen among those generated in the subproblems. However, the output from the Dantzig-Wolfe decomposition approach consists of an optimal convex combination of the different plans. For achieving optimality, a branch and bound approach or at least a limited tree search must be applied on some suitable characteristic of the problem. Such a characteristic can concern the exact time period a customer should be visited. Concerning a limited tree search, one may implement a approach of fixing more and more of the production and transportation plans until all parts of the plans are decided, see [12] for an example.

\section{Enhanced Agent-based Decisions by De- composition}

The basis for the planning of the studied problem is the agent-based decision making system, presented in Figure 12. For this system to strive towards system optimality, suitable requests and answers need to be sent between the agents. Information provided by the decomposition approach can assist in this process. Either such information can be obtained after termination of the decomposition but more efficiently during the progress of the process.

In particular, the transport chain coordinator agent can from the coordinator problem obtain information about current best offers of production and transportation. Also, the agents can receive, by the dual prices, approximate information about production cost of the different products $(\mu)$, cost of meeting customer demand at the different customers $(\lambda)$, and cost of transport resources $(\tau)$, for the different time periods. These prices are obtained from the decomposition approach (e.g. the Dantzig- 
Wolfe). Note that these dual prices represent values/costs from a system perspective of products or resources in different time periods. These dual prices creates credibility with respect the cost consequences to the whole system of the solution since alternative solution can approximately be evaluated by all agents which have access to these dual prices. Knowing the potential effect of changes to the plan at other part of the system, at least by approximate costs, can facilitate a beneficial utilization of flexibility (e.g. production can be set to occur earlier or later for matching transport requirements).

If the planning situation suddenly changes (e.g. new customer orders appear), the previous dual prices might be accurate enough for assisting the agent to find new good solutions.

Hence, we propose to run the agent-based decision making system in parallel with the Dantzig-Wolfe decomposition approach as outlined in Figure 5.

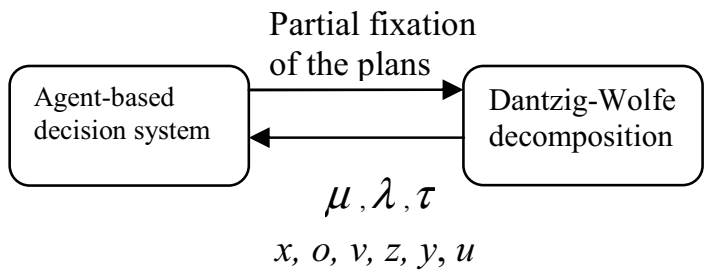

Figure 5 Interaction between the Agent-based decision system and the Dantzig-Wolfe decomposition approach.

In a basic setting, the agent-based decision system sends information on which fixations that are made in the plan. These fixations represent parts of the plans which not easily can be re-planned from a real planning perspective. In a more advanced version, it could send some potential but not necessary fixations and with different penalties for undoing the fixations, in order more thoroughly explore the potential improvements of currents solution/plan.

In the proposed basic setting of the Dantzig-Wolfe decomposition approach, it obeys the current fixations but ignore the combinatorial requirements. It generates new columns until new improving columns cannot be found. Whenever requested by the agent-based decision system, it delivers the currently best dual prices and plans. It may also suggest a few of the different extreme plans making up the best solution found so far. In a more advanced version, it may be useful within the Dantzig-Wolfe decomposition approach, to handle some or all of the combinatorial issues. Then some additional fixing of the plans could occur in the master and thereby potentially provide the agent-based decision system with solutions of higher quality.

The solution methods required for solving the subproblems can also be used in the agents of the decision making system. Then the agents can act, at least tempo- rally, more independently and is not dependent on other more advanced approaches.

\section{Conclusion and Future Work}

A decomposition approach has been suggested which should support the agent-based decision system. The decomposition approach should provide the decision system with useful partial plans (e.g. production or transportations). Further it should provide the system with prices or resources assisting the negotiations and increase the acceptance of locally deteriorating solution.

Based on experiences from [10] and [12] experiments can commence of the studied case. Then an interesting issue is to study different strategies for taking advantage of the information from the decomposition approach more closely. One may also test approaches for handling the planning within the decision system by mainly relying on local search approaches compared to the in this paper suggested approach based on decomposition.

\section{Acknowledgements}

This work is primarily carried out within the project "Integrated Production and Transportation Planning within Food Industry" (see www.ipd.bth.se/fatplan) with founding from the Swedish Knowledge Foundation (www.kks.se). Further VINNOVA, the Swedish Agency for Innovation Systems, is in part financially supporting this research.

\section{References}

[1] L.S. Lasdon. Optimization Theory for Large Systems, Macmillan. (1970)

[2] C. Barnhart, E. L. Johnson, G. L. Nemhauser, M. W. P. Savelsbergh and P. H. Vance, Branch-and-Price: Column Generation for Solving Huge Integer Programs, Operations Research, Vol. 46. (1998)

[3] L.A. Wolsey, Integer Programming, Wiley. (1998)

[4] U.S. Karmarkar and L. Schrage. The deterministic dynamic product cycling problem, Operations Research, Vol. 33, 326345. (1985)

[5] P. Brandimarte and A. Villa, Advanced Models for Manufacturing Systems Management, CRC Press, Inc. (1995)

[6] L.A. Wolsey, MIP modelling of changeovers in production planning and scheduling problems, European Journal of Operational Research, Vol. 99. (1997)

[7] M. Wooldridge: An Introduction to MultiAgent Systems, Wiley, ISBN: 0-471-49691-x (2002)

[8] H.V.D. Parunak. Industrial and Practical Applications of DAI, Multiagent Systems, (editor G. Weiss). MIT Press (1999) 
[9] P. Davidsson, S.J. Johansson, J.A. Persson, and F. Wernstedt, "Agent-based Approaches and Classical Optimization Techniques for Dynamic Distributed Resource Allocation: A preliminary study", AAMAS'03 workshop on Representations and Approaches for Time-Critical Decentralized Resource/Role/Task Allocation, Melbourne, Australia, (2003).

[10] M. Bergkvist, P. Davidsson, J.A. Persson, and L. Ramstedt "A Hybrid Micro-Simulator for Determining the Effects of Governmental Control Policies on Transport Chains" Joint AAMAS Workshop on Multi-Agent and Multi-Agent-Based Simulation, New York City, (2004)
[11] L.A. Wolsey, Integer Programming, Wiley. (1998)

[12] Persson, J.A., Göthe-Lundgren, M., "Shipment Planning at Oil Refineries Using Column Generation and Valid Inequalities", European Journal of Operational Research, Article In Press, (2004) 